\title{
The probability of inflation in Loop Quantum Cosmology
}

\section{William Nelson*iKing's College London \\ E-mail: william.nelsonekcl.ac.uk}

\begin{abstract}
The probability of there being sufficient inflation to solve the fine-tuning associated with the horizon and flatness problems has recently been shown to be exponentially small, within the context of classical general relativity. Here this result is extended by considering loop quantum gravity effects, that are significant at small scales. In addition to accounting for high-energy departures from classicality, it is shown that, in contrast to the classical case, the loop quantum cosmological probability measure is naturally finite, at least in some well defined region. It is also shown that these loop quantum gravity corrections can overcome the classical suppression of the probability only for extremely unnatural choices of ambiguity parameters, implying that single field, slow-roll inflation is exponentially unlikely.
\end{abstract}

From Quantum to Emergent Gravity: Theory and Phenomenology

June 11-15 2007

Trieste, Italy

\footnotetext{
*Speaker.

†This work was done in collaboration with Cristiano Germani and Mairi Sakellariadou [ef]
} 


\section{Introduction}

Cosmological inflation is the most popular method of explaining the fine-tuning difficulties associated with the standard hot big bang model [2] (although many other models exist [3, 4 , 51). Inflation is essentially an accelerated era of expansion that took place in the early universe, and it is easily motivated by the basic principles of general relativity and quantum field theory. Despite its many successes, inflation can only be said to solve the fine tuning problem if its onset is independent of the initial conditions. However the fact that a quantum theory of gravity has yet to be fully developed means that even when this issue is addressed, robust conclusions cannot be drawn [6, 7]. This issue of initial conditions can be overcome by using a measure based on the dynamical structure of the theory and assuming some late time equivalence between distinct universes. This was the method used in Ref. [8], where it was shown that the probability of a universe at the end of an inflationary phase having had $N$ or more efoldings of single field, slow-roll inflation is proportional to $\exp (-3 N)$. Since $N$ is typically required to be approximately 60 , this would imply that the onset of inflation needs to be significantly fine-tuned, however the calculation is dependent on the equations of classical general relativity being valid at all scales. In addition there is a restriction on types of inflationary potential that are amenable to this approach, that was not discussed by the authors of Ref. [8].

Here the effect of the quantum gravity in the early universe will be accounted for by considering corrections arising from loop quantum cosmology. The probability of having $N$ or more efoldings of inflation within this setting will be investigated and compared to the classical result and precise constraints on the validity of this method will be discussed. A more detailed derivation and discussion can be found in Ref. [1].

\section{Loop Quantum Cosmology}

Loop quantum gravity [9, 10] is a background independent, non-perturbative quantisation of general relativity, which has been shown to be well behaved at classical singularities such as the big bang. Despite its successes the full theory, in an inhomogeneous setting is not entirely understood and in many cases it is not even possible to define a continuum limit. By restricting the symmetries of the theory however, it is possible to produce a well defined classical limit. In particular we are interested in homogeneous, isotropic cosmologies, which make the theory tractable. To help ensure that the symmetry reduction doesn't induce additional effects, wherever possible, the derivation follows that of the full theory [11] (see also Martin Bojowald's contribution to these proceedings). A brief introduction to LQC is given below and follows the notation given in [12, 13. For a more complete description of the formalism see [14].

Loop quantum cosmology (LQC) formulates general relativity in terms of Ashtekar variables: $S U(2)$ valued triads $E_{\mathrm{i}}^{a}$ and a $S U(2)$ connection $A_{\mathrm{i}}^{a}$ (where ijk etc. are $S U(2)$ indices, whilst $a b c$ etc. are coordinate indices). The quantisation procedure uses holonomies of $A_{\mathrm{i}}^{a}$ along a specified edge $e$,

$$
h_{e}(A)=\mathscr{P} \exp \int_{e} \dot{\gamma}^{a}(s) A_{a}^{\mathrm{i}}(\gamma(s)) \tau_{\mathrm{i}} d s,
$$

where $\mathscr{P}$ infers path ordering on the exponential, $\dot{\gamma}^{a}$ is the tangent vector along the edge $e$, and $\tau_{i}$ are the basis of the $S U(2)$ Lie algebra. Restricting to isotropic, homogeneous systems means we 
need only consider straight edges along the integral curves of the basis vectors $X_{\mathrm{i}}^{a}$. In this case the connection is given by a (dynamic) multiple of the basis one forms $\omega_{a}^{\mathrm{i}}, A_{a}^{\mathrm{i}}=\tilde{c}(t) \omega_{a}^{\mathrm{i}}$, whilst the triad is $E_{\mathrm{i}}^{a}=\sqrt{{ }^{0} g} \tilde{p}(t) X_{\mathrm{i}}^{a}$, with ${ }^{0} g$ is the determinant of the fiducial metric ${ }^{1}$. With this the holonomies becomes simply,

$$
\begin{aligned}
h_{\mathrm{i}}(A) & =\exp \left[\frac{-i \mu_{0} \sigma_{\mathrm{i}}}{2} \tilde{c}\right] \\
& =\cos \left(\frac{\mu_{0} \tilde{c}}{2}\right)+2 \tau_{\mathrm{i}} \sin \left(\frac{\mu_{0} \tilde{c}}{2}\right),
\end{aligned}
$$

where $\sigma_{\mathrm{i}}$ are the Pauli matrices and $\tau_{\mathrm{i}}=-i \sigma_{\mathrm{i}} / 2$ are the basis of the $\mathrm{SU}(2)$ Lie algebra and $\mu_{0}$ is the orientated length of the edge with respect to the fiducial metric. The choice of $\mu_{0}$ is arbitrary [14] and will now be set to unity. When formulated in these terms the evolution equation (the Hamiltonian constraint) is a discrete equation, with a discreteness scale given by $a_{\mathrm{Pl}}=\sqrt{\gamma / 6} l_{\mathrm{Pl}}$, where $l_{\mathrm{Pl}}$ is the Planck length and $\gamma$ is the Barbero-Immirzi parameter. This ambiguity comes from the definition of the connection in terms of standard ADM variables (or to put it another way, an ambiguity in formulating GR in terms of triads and connections), $A_{\mathrm{a}}^{i}=\Gamma_{\mathrm{a}}^{i}+\gamma K_{\mathrm{a}}^{i}$, where $K_{\mathrm{a}}^{i}$ is the extrinsic curvature one form and $\Gamma_{\mathrm{a}}^{i}$ is the spin connection. Calculations of black hole entropy[16, 17] fixes this to be $\gamma \approx .02735$. We will be interested only in effective, continuous equations which are valid for length scales $a \gg a_{\mathrm{Pl}}$.

Classically the canonical variables $\tilde{c}, \tilde{p}$ are related through

$$
\{\tilde{c}, \tilde{p}\}=\frac{\kappa \gamma}{3 V_{0}},
$$

where $\kappa \equiv 8 \pi G$ and $V_{0}$ is the volume of the fiducial cell $\mathscr{V}$ as measured by the fiducial metric. Defining

$$
p=V_{0}^{2 / 3} \tilde{p} \quad \text { and } \quad c=V_{0}^{1 / 3} \tilde{c},
$$

with the triad component $p$ determining the physical volume of the fiducial cell, and the connection component $c$ determining the rate of change of the physical edge length of the fiducial cell, one obtains

$$
\{c, p\}=\frac{\kappa \gamma}{3},
$$

independent of the volume of the fiducial cell.

By analogue with the full theory the kinematic Hilbert space is extended via the Bohr compactification of the real line [14]. An orthonormal basis for this Hilbert space is $\{|\mu\rangle\}$, where

$$
\langle c \mid \mu\rangle=e^{i \frac{\mu c}{2}}
$$

The triad operator acts on these basis states as

$$
\hat{p}|\mu\rangle=-i \frac{\kappa \gamma \hbar}{3} \frac{\partial}{\partial c}|\mu\rangle=\frac{\kappa \gamma \hbar}{6} \mu|\mu\rangle,
$$

\footnotetext{
${ }^{1}$ This fiducial metric is a complication that arises only for open universe and is used to define the volume to which integral are restricted to ensure they remain finite. Clearly physical results must not depend on this volume[ [15].
} 
Clearly the volume operator $\hat{V} \equiv \hat{a}^{3}=|\hat{p}|^{3 / 2}$ also has eigenstates in this basis,

$$
\hat{V}|\mu\rangle=V_{\mu}|\mu\rangle=\left(\frac{\kappa \gamma \hbar|\mu|}{6}\right)^{3 / 2}|\mu\rangle
$$

To calculate the eigenvalues of the inverse volume operator, the classical expression [18],

$$
p^{-1}=\left(p^{L-1}\right)^{1 /(L-1)}=\left(\frac{3}{\kappa \gamma L}\left\{c, p^{L}\right\}\right)^{1 /(L-1)},
$$

is used. This is a classical identity independent of $\mathrm{L}$, but will be quantized to different operators for different L. Thus, L plays the role of a quantization ambiguity. After quantisation the inverse volume operator acts as [19]

$$
\widehat{V^{-1}}|\mu\rangle=\left(\frac{9}{\kappa \gamma \hbar L J(J+1)(2 J+1)} \sum_{m=-J}^{J} m V^{2 L / 3}\left(\mu+2 m \mu_{0}\right)\right)^{3 / 2(1-L)}|\mu\rangle .
$$

The eigenvalue can be approximated by a continuous function [20] $d_{J, L}(a)=D_{L}(q) a^{-3}$, where $q=a^{2} / a_{\star}^{2}$ and $a_{\star}^{2}=\gamma l_{\mathrm{pl}}^{2} J / 3$. The function $D_{L}(q)$ (see figure (1) ) accounts for the difference between the LQC and the classical inverse volume eigenvalues,

$$
\begin{aligned}
D_{L}(q)= & \left(\frac { 3 q ^ { 1 - L } } { 2 L } \left[\frac{1}{L+2}\left((q+1)^{L+2}-|q-1|^{L+2}\right)\right.\right. \\
& \left.\left.-\frac{q}{1+L}\left((q+1)^{L+1}-\operatorname{sgn}(q-1)|q-1|^{L+1}\right)\right]\right)^{3 /(2-2 L)}
\end{aligned}
$$

The Hamiltonian constraint is given by [19],

$$
{ }^{(J)} \hat{\mathscr{C}}_{\mathrm{g}}=\frac{3 i \operatorname{sgn}(|p|)}{\kappa^{2} \hbar \gamma^{3} J(J+1)(2 J+1)} \sum_{i j k} \varepsilon^{i j k} \operatorname{tr}\left(\hat{h}_{i} \hat{h}_{j} \hat{h}_{i}^{-1} \hat{h}_{j}^{-1} \hat{h}_{k}\left[\hat{h}_{k}^{-1}, \hat{V}\right]\right),
$$

which can be made self-adjoint simply by symmetrising,

$$
{ }^{(J)} \hat{\mathscr{H}}_{\mathrm{g}}=\frac{1}{2}\left({ }^{(J)} \hat{\mathscr{C}}_{\mathrm{g}}+{ }^{(J)} \hat{\mathscr{C}}_{\mathrm{g}}^{\dagger}\right) .
$$

The action of this Hamiltonian constraint on the basis states $|\mu\rangle$ results in a complicated difference equation of order $8 J$, however it is well approximated by the equation [19]

$$
\mathscr{H}=-3 a \dot{a}^{2}+s(a) \mathscr{H}_{\phi}=0,
$$

where $s(a)=a S\left(a^{2} / a_{\mathrm{G} \star}^{2}\right)$ is a continuous function that approximates the corrections to gravity terms, with $a_{\mathrm{G} \star}$ given by $a_{\mathrm{G} \star}=\gamma l_{\mathrm{Pl}} J_{\mathrm{G}} / 3$. Defining $q_{\mathrm{G}}=a^{2} / a_{\mathrm{G} \star}^{2}$ the function is (see figure. (2)),

$$
S\left(q_{\mathrm{G}}\right)=\frac{4}{\sqrt{q_{\mathrm{G}}}}\left(\frac{1}{10}\left(\left(q_{\mathrm{G}}+1\right)^{5 / 2}+\operatorname{sgn}\left(q_{\mathrm{G}}-1\right)\left|q_{\mathrm{G}}-1\right|^{5 / 2}\right)-\frac{1}{35}\left(\left(q_{\mathrm{G}}+1\right)^{7 / 2}-\left|q_{\mathrm{G}}-1\right|^{7 / 2}\right)\right),
$$




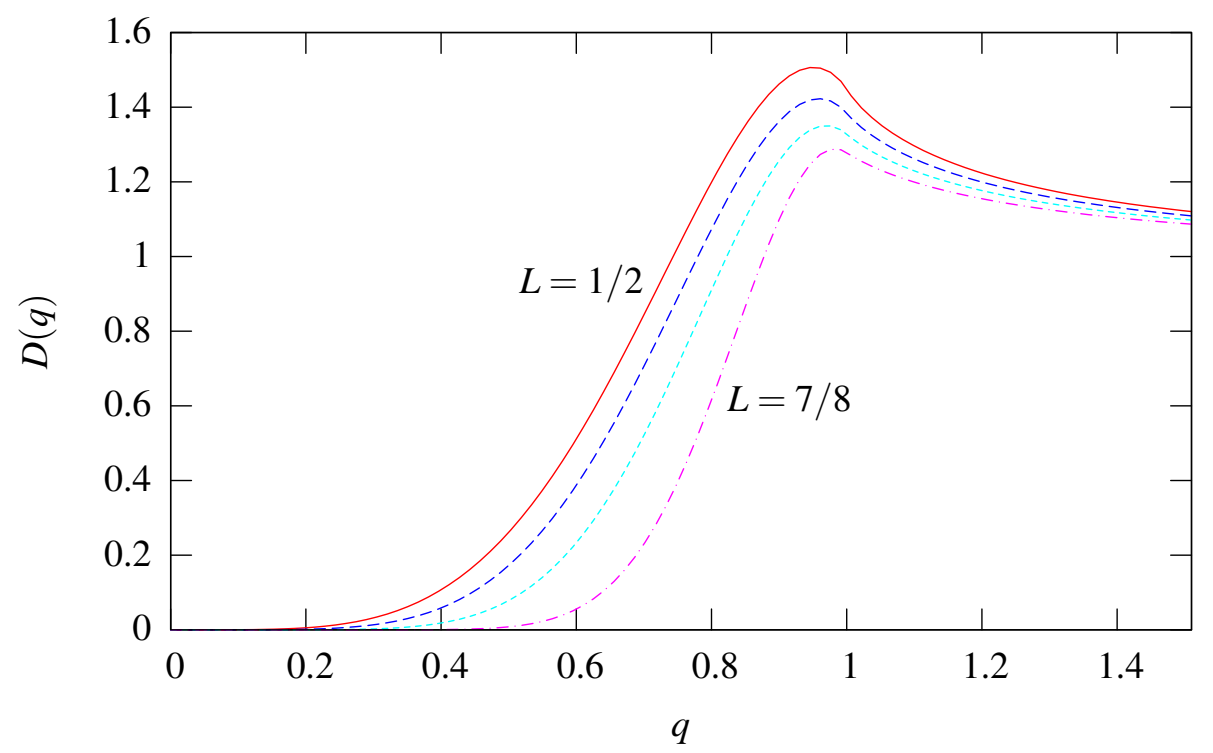

Figure 1: The functions $D_{1 / 2}(q), D_{5 / 8}, D_{3 / 4}$ and $D_{7 / 8}$ are plotted, clearly the quantisation ambiguity, $L$, has no qualitative effect on the effective equations.
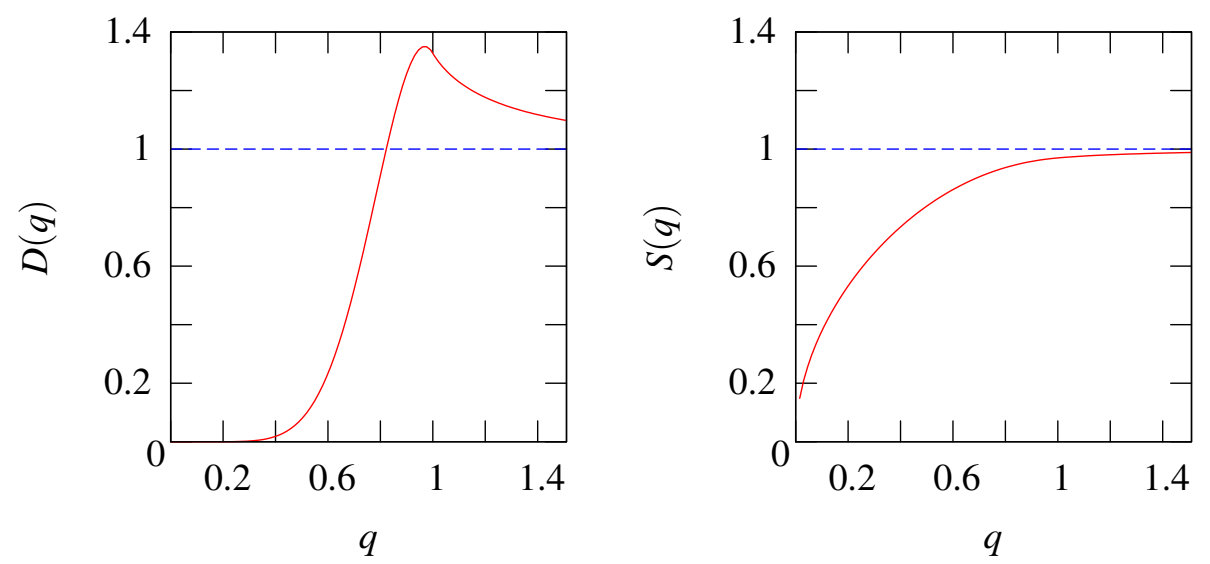

Figure 2: The function $D(q)$ (left) (with $L=3 / 4$ ) is the continuum approximation of the discrete quantum corrections to the classical inverse volume eigenvalues, whilst $S(q)$ (right) is the continuum approximation of the quantum corrections to the gravitational part of the Hamiltonian constraint. 
where $L$ is set to $L=3 / 4$ from now on, since it has no qualitative effects (see Figure (11)). Notice also that $J_{\mathrm{G}}$ is not necessarily the same as $J$ and that these effective equations are valid only in the continuum era, when $a \gg a_{\mathrm{Pl}}$.

Here the matter component we are concerned with is the inflaton, for which we have [21],

$$
\mathscr{H}=-\left(\frac{\dot{a}}{a}\right)^{2}+\frac{\kappa S\left(q_{\mathrm{G}}\right)}{3}\left[\frac{1}{2} D^{-(n+1)} \dot{\phi}^{2}+D^{m} V(\phi)\right]=0,
$$

where $V(\phi)$ is the inflaton potential and $m, n$ are further quantisation ambiguities arising due the fact that $\hat{V} \widehat{V^{-1}}$ is not unity ${ }^{2}$. Defining $H \equiv \dot{a} / a$, we arrive at the effective, loop quantum cosmological Friedmann equation,

$$
H^{2}=\frac{\kappa S\left(q_{\mathrm{G}}\right)}{3}\left[\frac{1}{2} D^{-(n+1)} \dot{\phi}^{2}+D^{m} V(\phi)\right] .
$$

To simplify the following calculation, $S\left(q_{\mathrm{G}}\right)$ will be set to unity, which is equivalent to choosing $J_{\mathrm{G}}=1 / 2$. More general choices will be discussed in at the end of section (6). Once again it is important to note that these effective equations are valid only for $a \gg \sqrt{\gamma} l_{\mathrm{Pl}}$ and $H^{-1} \ll \sqrt{\gamma} l_{\mathrm{Pl}}$. These limits are crucial in the calculation of the measure on the space of solutions.

The dynamics of the scalar field are given by the conservation equation [21],

$$
\ddot{\phi}+\left[3 H-(1+n) \frac{\dot{D}_{l}}{D_{l}}\right] \dot{\phi}+D_{l}^{m+n+1} V^{\prime}(\phi)=0,
$$

where $V^{\prime} \equiv \partial V / \partial \phi$

\section{Cosmological Measures}

To be able to calculate probabilities, a measure on the space of solutions of the theory must be defined. Without a full theory of quantum gravity to produce such a measure on the initial conditions there is a significant ambiguity in how it is defined. Despite this any measure must satisfy certain basic properties [22]:

1. it must be positive definite (and finite),

2. it must not depend on the choice of variables used,

3. it must respect all the symmetries of the phase space.

The necessity of the first point is self-evident if the measure is to produce sensible probabilities. Loosely speaking the second point says that the probability of a universe should be independent of the time at which we choose to calculate it, whilst the third forbids the introduction of any ad hoc cutoffs into the theory.

The importance of placing a measure on the different possible universes is crucial to modern cosmology since fundamental theories are no longer expected to provide a unique cosmological solution. Thus the key question in cosmology is, 'how likely is our universe?'. It is well known

\footnotetext{
${ }^{2}$ In fact it has been shown [19] that the effective Hamiltonian should include a correction term that induces a bounce at small scales. In what follows, such scales are not reached and the Hamiltonian given here is a good approximation.
} 
that classical general relativity (and the observed expansion) predict an initial big bang singularity and it is largely excepted that the full theory of quantum gravity will overcome this breakdown in predictability. Because such candidate theories (e.g. LQG, M-theory etc.) typically differ from classical physics only at the highest energy scales, it is only through cosmology that they can, in principle be tested. However any signatures imprinted on the universe by these non-classical effects are typically expected to be extremely small and so the prediction that universes such as our own are a 'generic' outcome is usually taken evidence in favour of the theory. However without a measure on the possible solutions any discussion on how likely a particular outcome is, at best speculative.

Inflation is the archetypal example and has produced much debate on whether or not its onset is fine tuned [23, 24, 25]. Since the purpose of introducing an inflationary epoch into the early universe was to eliminate the fine tuning problems associated with the flatness and horizon problems, it can only be said to have succeeded if it occurs without similar fine tuning. Without a fundamental theory that predicts inflation, the issue of its generality can only be tackled by introducing a measure on the cosmological histories that contain inflation. The canonical measure proposed by Gibbons, Hawking and Stewart [22] was shown to be infinite [26], however more recently Gibbons and Turok [8] demonstrated that the measure can be regularised and that the results (for certain quantities) are robust. The measure is derived in the next section, however in the case of LQC the regularisation procedure is not required and so this won't be discussed.

It is important to note that whilst it has turned out to be difficult to produce a different measure that satisfy all the criteria given above, there is nothing to say that it cannot be done. This means that results derived with this measure cannot be directly compared to results derived using a different measure, although it may be hoped that certain properties within a theory are largely measure independent.

\section{The Measure}

The canonical cosmological measure of Ref. [22] is based on the Hamiltonian structure of a theory and satisfies all the required criteria (although it is by no means unique [27]) and is the one used here.

As with all phase spaces, that associated with cosmology has a symplectic form,

$$
\Omega=\sum_{i=1}^{k} \mathrm{~d} P_{i} \wedge \mathrm{d} Q^{i}
$$

where $Q_{i}$ and $P_{i}$ are the dynamical degrees of freedom and their conjugate momenta, respectively. The $k^{\text {th }}$ power of this gives the volume element of the space. The Hamiltonian constraint restricts the physical trajectories to lie on a $(2 k-1)$-dimensional surface $M$, of the full phase space, which is what is known as the multiverse. $M$ also contains a closed symplectic form $\omega=\sum_{i=1}^{k-1} \mathrm{~d} P_{i} \wedge \mathrm{d} Q^{i}$, related to $\Omega$ via,

$$
\Omega=\omega+\mathrm{d} \mathscr{H} \wedge \mathrm{d} t \Rightarrow \omega=\left.\Omega\right|_{\mathscr{H}=0} .
$$

This measure can be produced from the Hamiltonian given in Eq. (2.16), which includes the effective quantum gravity corrections to the classical equations. 


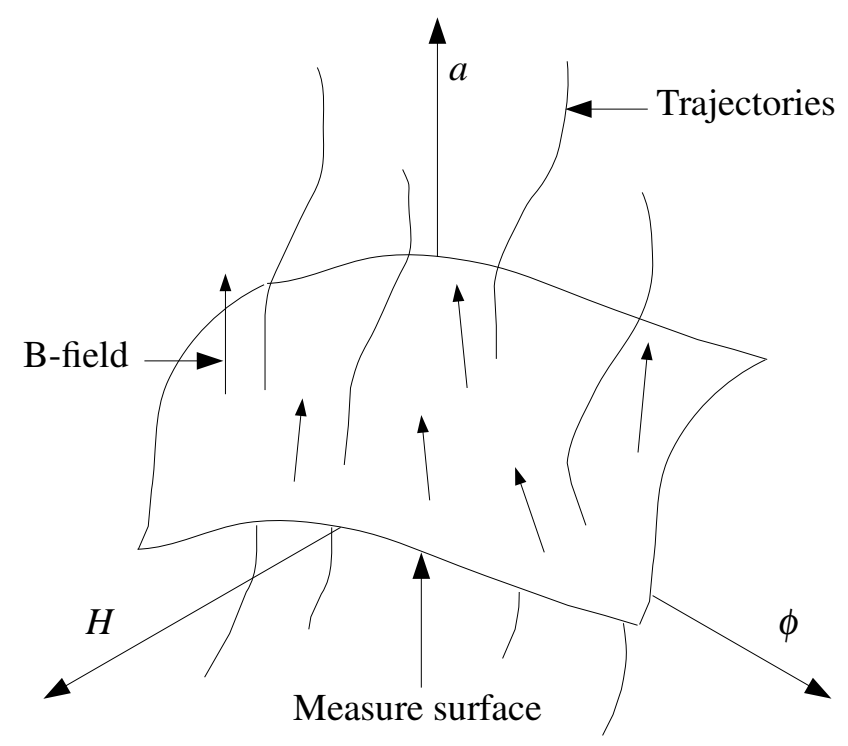

Figure 3: The probability measure is defined by integrating the $\mathbf{B}$ field over a constant surface in the 3dimensional phase space produced by using the Hamiltonian constraint to eliminate one of the dynamical variables.

In this case (a FLRW universe containing only a scalar field) there are only two canonical variables $(a, \phi)$ and so $k=2$. A divergence-less field $\mathbf{B}$ can be defined, given by

$$
B_{a} \equiv \frac{1}{2} \varepsilon_{a b c} \omega_{b c}
$$

where $\varepsilon_{a b c}$ is totally anti-symmetric and $a, b, c$ go from 1 to 3 . The flow of trajectories in the phase space across a surface is given by $\mathbf{B}$ evaluated on that surface and this is used to define the measure $\mathscr{N}$,

$$
\mathscr{N}=\int \mathbf{B} \cdot \mathrm{d} \mathbf{S}
$$

where $\mathbf{S}$ is an open surface crossing each trajectory only once. The scheme is depicted in Figure (3).

By construction $\mathbf{B}$ is divergence-less and so a vector potential can be defined $\mathrm{d} \mathbf{A}=\mathbf{B}$. Then using Stoke's theorem the measure becomes,

$$
\mathscr{N}=\oint \mathbf{A} \cdot \mathrm{d} \mathbf{l},
$$

where $\mathbf{l}=\partial \mathbf{S}$ is the boundary of $\mathbf{S}$. This ensures that the measure depends only on the flux of trajectories through $\mathbf{I}$ and is independent of the (topologically equivalent) surface chosen.

\section{Total volume of phase space}

Once a measure on the phase space has been defined the probability of a particular set of trajectories can be calculated as the ratio of the measure of those trajectories to the measure of the total phase space. In the classical case this is not well defined [26] due to a divergence in the total 
phase space measure, unless a small curvature cut-off is introduced [8]. The effective equations of LQC remove this divergence, since there is a minimum scale that can be probed within this formalism, $a \gg \sqrt{\gamma} l_{\mathrm{Pl}}$. However there is a further possible source of divergence in the classical case that is not removed by the LQC corrections, that restricts the form of inflationary potential that can be used.

Here a sketch of calculation is given, the details being given in [1]. The measure of the total phase space, defined by Eqs. (2.16), (2.18) can be calculated using the momenta associated with the scale factor and the scalar field $\phi$,

$$
P_{a}=-6 a^{2} H, P_{\phi}=a^{3} D_{l}^{-(n+1)} \dot{\phi} .
$$

In terms of $q=a^{2} / a_{\star}^{2}$ and using Eq. (2.17) with $S\left(q_{\mathrm{G}}\right)=1$, the measure evaluated on a $q=q_{\mathrm{s}}$ surface, where $q_{\mathrm{s}}$ is a constant gives,

$$
\mathscr{N}=-\frac{3}{4 \pi G} \iint \frac{H a_{\star}^{3} q_{\mathrm{s}}^{\frac{3}{2}} D_{\mathrm{s}}^{-\left(\frac{n+1}{2}\right)}}{\sqrt{\frac{3 H^{2}}{4 \pi G}-2 D_{\mathrm{s}}^{m} V}} \mathrm{~d} H \mathrm{~d} \phi
$$

where from now on the $l$ label is dropped and the notation $f\left(a_{\mathrm{s}}\right)=f_{\mathrm{s}}$ is used.

The $H$ integral can be performed to give,

$$
\mathscr{N}=-a_{\star}^{3} q_{\mathrm{s}}^{\frac{3}{2}} D_{\mathrm{s}}^{-\frac{n+1}{2}} \int_{\phi_{\mathrm{i}}}^{\phi_{\mathrm{f}}} \sqrt{\frac{3}{4 \pi \gamma l_{\mathrm{Pl}}^{4}}-2 D_{\mathrm{s}}^{m} V(\phi)} \mathrm{d} \phi,
$$

where $\left[\phi_{\mathrm{i}}, \phi_{\mathrm{f}}\right]$ is the range on $\phi$ which ensures $\mathscr{N}$ is real.

It is clear Eq. (5.3) is by not always convergent, as was assumed in Ref. [8], in the classical $(D \rightarrow 1)$ case for potentials with only one minima. However it does converge for many physically interesting potentials (e.g. single minima potentials that are unbounded above). In the following sections specific potentials will be used to calculate probabilities. For the moment however all that is required is that the potential is such that Eq. (5.3) converges.

\section{Probability}

To calculate the probability of inflation, the measure of only the inflationary trajectories is needed i.e.

$$
\left.\mathscr{M} \equiv \mathscr{N}\right|_{\text {inflation }} .
$$

Using Stoke's theorem for the path $H=H_{\mathrm{s}}=$ const. on Eq. (5.2) restricted to only inflationary trajectories, we have

$$
\mathscr{M}=-\left.\oint\left|P_{\phi}\right| \mathrm{d} \phi\right|_{\text {inflation }},
$$

which is positive as inflation runs from higher to lower values of the scalar field $\phi$. Details of how this can be calculated and how the calculation differs from the classical case can be found in Ref. [1]. The result is

$$
\mathscr{M}=\frac{a_{\star}^{3} q_{\mathrm{s}}^{\frac{3}{2}}}{4 \pi G} D_{\mathrm{s}}^{-\left(\frac{n-m+1}{2}\right)} \delta \varepsilon_{\mathrm{i}} \exp \left(-3\left|1-(n-m+1) \frac{q_{\mathrm{s}}}{3 D_{\mathrm{s}}}\left(\left.\frac{\partial D}{\partial q}\right|_{\mathrm{s}}\right)\right| N\right),
$$


where

$$
\begin{aligned}
\delta \varepsilon_{\mathrm{i}} & \approx \frac{1}{12 A_{\mathrm{s}}^{3}} \sqrt{\frac{V\left(\phi_{\mathrm{i}}\right)}{24 \pi G}}\left(\left.\frac{1}{V\left(\phi_{\mathrm{i}}\right)} \frac{\partial V(\phi)}{\partial \phi}\right|_{\mathrm{i}}\right)^{2}, \\
A & \equiv\left|1-(n-m+1) \frac{q}{3 D} \frac{\partial D}{\partial q}\right| D^{-(n+1) / 2},
\end{aligned}
$$

and the constant $q=q_{s}$ surface on which the measure is being calculated is taken to be at the end on inflation i.e. at the breakdown of the slow-roll conditions. To be able to evaluate this a potential must be given and here potentials of the form,

$$
V(\phi)=\frac{\mu^{4}}{2 \alpha !}\left(\frac{\phi}{\mu}\right)^{2 \alpha}
$$

are considered. Notice that these belong to the class of potentials that make Eq. (5.3) finite and so well defined probabilities can be produced. Using the slow-roll approximation (and the assumption that $\phi_{\mathrm{f}} \ll \phi_{\mathrm{i}}$ ) with this class of potentials, Eq. (6.4), can be shown to be,

$$
\delta \varepsilon_{\mathrm{i}} \approx \frac{l_{\mathrm{Pl}}^{-1}}{\sqrt{2 \alpha !}} \frac{\alpha^{2}}{3 A_{\mathrm{s}}^{3} \sqrt{24 \pi}}\left(\frac{\mu}{\phi_{\mathrm{i}}}\right)^{2-\alpha}
$$

where

$$
\phi_{\mathrm{i}} \approx\left(\frac{\alpha}{4 \pi G} \int_{a_{\mathrm{i}}}^{a_{\mathrm{s}}} \frac{D^{n+1}}{\left[1-(n-m+1) \frac{q}{3 D} \frac{\partial D}{\partial q}\right]} \frac{\mathrm{d} a}{a}\right)^{1 / 2} .
$$

Notice that for $\alpha=1$ and $D=1$ we recover the standard GR result $\phi_{\mathrm{i}} \approx \sqrt{N /(4 \pi G)}$.

Finally, from Eqs. (5.3), (6.3) and (6.7), the probability $\mathscr{P}(N)$ of having $N$ e-folds of slow-roll inflation is

$$
\mathscr{P}(N) \approx \beta^{2}\left(\frac{\mu}{\phi_{\mathrm{i}}}\right)^{2-\alpha}\left(l_{\mathrm{P} 1} \mu\right)^{\frac{2-\alpha}{\alpha}} \exp \left(-3\left|1-(n-m+1) \frac{q_{\mathrm{s}}}{3 D}\left(\left.\frac{\partial D}{\partial q}\right|_{\mathrm{s}}\right)\right| N\right)
$$

where

$$
\beta^{2}=\frac{\alpha^{3}}{144}\left[\frac{2}{3 \pi(2 \alpha !)}\right]^{\frac{\alpha+1}{2 \alpha}} 2^{\frac{\alpha+2}{2 \alpha}} \pi^{\frac{\alpha-1}{\alpha}} \gamma^{\frac{\alpha+1}{2 \alpha}} \frac{\Gamma\left(\frac{3 \alpha+1}{2 \alpha}\right)}{\Gamma\left(\frac{1}{2 \alpha}\right)} D_{s}^{m \frac{\alpha+1}{2 \alpha}} A_{s}^{-3}
$$

This changes qualitatively for renormalisable $(\alpha \leq 2)$ and non-renormalisable $(\alpha>2)$ potentials and here we concentrate only on renormalisable potentials [28] i.e. $\alpha=1,2$.

The above calculation can be repeated using $S\left(q_{\mathrm{G}}\right) \neq 1$ to give

$$
\mathscr{P}(N) \approx \beta^{2}\left(\frac{\mu}{\phi_{\mathrm{i}}}\right)^{2-\alpha}\left(l_{\mathrm{P} 1} \mu\right)^{\frac{2-\alpha}{\alpha}}\left[S\left(\frac{a^{2}}{a_{G}^{2}}\right)\right]^{\frac{\alpha+4}{4 \alpha}} \times \exp \left(-3\left|1-(n-m+1) \frac{q_{\mathrm{s}}}{3 D}\left(\left.\frac{\partial D}{\partial q}\right|_{\mathrm{s}}\right)\right| N\right),
$$

where now,

$$
\phi_{\mathrm{i}} \approx\left(\frac{\alpha}{4 \pi G} \int_{a_{\mathrm{i}}}^{a_{\mathrm{s}}} \frac{D^{n+1}}{S\left(\frac{a^{2}}{a_{G}^{2}}\right)\left[1-(n-m+1) \frac{q}{3 D} \frac{\partial D}{\partial q}\right]} \frac{\mathrm{d} a}{a}\right)^{1 / 2} .
$$

Since $S\left(q_{\mathrm{G}}\right)<1$ it is clear that choosing $j_{\mathrm{G}} \neq 1 / 2$ (i.e. $S \neq 1$ ) slightly reduces the probability of inflation. However, for $a_{\mathrm{i}}>a_{\mathrm{G}}, S\left(q_{\mathrm{G}}\right)$ is, well approximated by 1 , thus the conclusions for the $j_{\mathrm{G}}=1 / 2$ case remain qualitatively unchanged. Therefore in the following we shall only consider the $j_{\mathrm{G}}=1 / 2$ fundamental representation case. 


\section{Evaluating the probability}

Whilst Eq. 6.10 looks much more complicated than the classical result given in Ref. [8], it can be simplified significantly. In order to have sufficient inflation to solve the standard cosmological problems, approximately 60 efoldings is required i.e.

$$
e^{60} \approx \frac{a_{\mathrm{s}}}{a_{\mathrm{i}}}<\frac{a_{\mathrm{s}}}{a_{\mathrm{Pl}}},
$$

where $a_{\mathrm{Pl}}$ is the minimal scale which can be probed by the effective equations of LQC used here. The scale at which quantum effects become significant, $a_{\star}$, is governed by $j$. This ambiguity has been restricted by particle physics experiments to be $j<10^{20}$, from which it is easy to see that $a_{\mathrm{s}} \gg a_{\star}$ (i.e. inflation ends well into the classical epoch), allowing functions evaluated on $a_{\mathrm{s}}$ to be expanded in the $q \gg 1$ limit.

The first term in the probability, Eq. (6.10) that is of interest is the exponential suppression factor

$$
\exp \left(-\left.3\left[1-(n-m+1) \frac{q}{3 D} \frac{\partial D}{\partial q}\right]\right|_{a_{\mathrm{s}}} N\right)
$$

It seems possible to overcome the classical exponential suppression simply by choosing $n, m$ and $a_{\mathrm{S}}$ so that $\left.\left[1-(n-m+1) \frac{q}{3 D} \frac{\partial D}{\partial q}\right]\right|_{a_{\mathrm{s}}} \sim \mathscr{O}(0)$. However the probability is also proportional to

$$
\beta^{2} \propto\left[\left.\left(1-(n-m+1) \frac{q}{3 D} \frac{\partial D}{\partial q}\right)\right|_{a_{\mathrm{s}}}\right]^{3}
$$

Therefore the highest probability is found when $\left.\left[1-(n-m+1) \frac{q}{3 D} \frac{\partial D}{\partial q}\right]\right|_{q} \sim \mathscr{O}(1)$. Thus the exponential suppression present in the classical $(D \rightarrow 1)$ case found in Ref. ([i] $)$ is not removed.

The most interesting term is $\mu / \phi_{\mathrm{i}}$, since $\phi_{\mathrm{i}}$ contains an integral over all scales. Thus the behaviour of $\left[1-(n-m+1) \frac{q}{3 D} \frac{\partial D}{\partial q}\right]^{-1}$ throughout inflation must be investigated. In order to significantly increase the probability, there cannot be a zero of $\left[1-(n-m+1) \frac{q}{3 D} \frac{\partial D}{\partial q}\right]$ at any point during inflation, which implies that $a_{\mathrm{i}}$ has to be larger than the scales of any such zeros. It can be shown [1] that this factor does not significantly increase the probability (relative to the exponential suppression), for values $N \gtrsim 22.5$ (the 22.5 arises because of the experimental limits on $j$ and is only relevant for a very small range of $n-m+1)$.

Finally the probability contains the factor $\beta^{2} \propto D_{s}^{\frac{m(\alpha+1)-\alpha(n+1)}{2 \alpha}}$. Since $D>1$ at the end of inflation, for sufficiently large powers it is possible for this term to become large. However it can only overcome the exponential suppression for ambiguity parameters that satisfy

$$
\frac{m(\alpha+1)-\alpha(n+1)}{2 \alpha} \gtrsim 10^{110}
$$

Clearly this cannot be considered a natural choice.

\section{Conclusion and discussion}

Cosmological inflation can only be said to solve the many fine-tuning problems associated with the hot big bang model if it is a generic outcome of a theory. This problem has been faced 
in the past [6, 7] and has recently been highlighted by Ref. [8]. In that study it was shown that, with a particular choice of measure, the probability of having sufficient inflation is exponentially suppressed by the number of efolds. This is an important result and its validity and generality should be proven. One major difficulty with the calculation of Ref. [8] is that classical physics was assumed to be valid at all scales during inflation, however it is well known that quantum corrections can no longer be neglected during the early phases of inflation. Here (and in detail in Ref. [1]) it has been shown how to include these quantum corrections within the formalism of effective continuum loop quantum cosmology.

Applying the canonical measure proposed by Ref. [22] to the effective equations of loop quantum cosmology allowed the probability of having sufficient, single field slow-roll inflation to be calculated. The resulting probability was then examined for the phenomenologically important $V \sim \phi^{2}$ and $V \sim \phi^{4}$ inflationary scenarios. It was shown that this probability is exponentially suppressed for all but the most extreme values of ambiguity parameters. In particular the values $m=n=0$ that are typically used in the literature do not alleviate the exponential suppression. It is known [29] that LQC can produce an era of super-inflation, in which the scalar field is driven up its potential. Such an epoch would not satisfy the slow-roll approximation used here and so would not be counted in this probability. However in this era perturbation theory is unstable [30] and so to produce the observed CMB anisotropies 60 subsequent efoldings of standard inflation are required and it is this that our probability considers.

Our findings do not imply that inflation itself is improbable, what they do show is that, at least in the case of the semi-classical regime of loop quantum cosmology and therefore of general relativity, inflation is not probable with this particular measure. Whenever cosmological probabilities are discussed a measure needs to be defined and, as has been shown here, this can lead to vastly differing results. Previously analysis on the likelihood of inflation (see for example [24]) have used the prior that all initial conditions are equally likely, whilst here a late time equivalence of universes that resemble our own has been taken. In the former case inflation was shown to be an attractor solution, whilst in the latter it is not. Which prior you choose to assume is a matter of taste, however what is clear is that any conclusions drawn will be highly sensitive to that choice. The crucial point then is that inflation may not be as likely as previously assumed.

To produce a definitive result one has to address inflation in full quantum gravity, or in a string theory context, so as to fully understand the initial conditions of the universe. Without such a fundamental treatment results on the generality of inflation will invariably be dependent on the choice of measure.

\section{Acknowledgements}

This work has been supported by the European Science Foundation network programme "Quantum Geometry and Quantum Gravity".

\section{References}

[1] C. Germani, W. Nelson and M. Sakellariadou, On the onset of inflation in loop quantum cosmology, arXiv:gr-qc/0701172. 
[2] A. Guth, Inflationary universe: A possible solution to the horizon and flatness problems, Phys. Rev. D 23, 347 (1981).

[3] R. H. Brandenberger and N. Shuhmaher, The confining heterotic brane gas: a non-inflationary solution to the entropy and horizon problems of standard cosmology, JHEP 0601, 074 (2006).

[4] P. J. Steinhardt and N. Turok, A Cyclic Model of the Universe, Science 296, 1436 (2002).

[5] C. Germani, N. E. Grandi and A. Kehagias, A Stringy Alternative to Inflation: The Cosmological Slingshot Scenario, [arXiv:hep-th/0611246].

[6] T. Piran, On general conditions for inflation, Phys. Lett. B 181, 238 (1986); D. S. Goldwirth, Inhomogeneous initial conditions for inflation, Phys. Rev. D 43, 3204 (1991).

[7] E. Calzetta and M. Sakellariadou, Inflation in inhomogeneous cosmology, Phys. Rev. D 45, 2802 (1992); idem Semiclassical effects and the onset of inflation, Phys. Rev. D 47, 3184 (1993).

[8] G. W. Gibbons and N. Turok, The measure problem in cosmology, [hep-th/0609095].

[9] C. Rovelli, Quantum Gravity, Cambridge University Press, Cambridge UK, 2004.

[10] T. Thiemann, Lectures on Loop Quantum Gravity, Lect. Notes Phys. 631, 41 (2003).

[11] M. Bojowald, Loop quantum cosmology, [gr-qc/0601085].

[12] W. Nelson and M. Sakellariadou, Lattice Refining Loop Quantum Cosmology and Inflation, arXiv:0706.0179 [gr-qc].

[13] W. Nelson and M. Sakellariadou, Lattice Refining LQC and the Matter Hamiltonian, arXiv:0707.0588 [gr-qc].

[14] A. Ashtekar, M. Bojowald, J. Lewandowski, Mathematical structure of loop quantum cosmology, Adv. Theor. Math. Phys. 7233 (2003)

[15] K. Vandersloot, Loop quantum cosmology, Ph.D. Thesis, Pennsylvania State University (2006).

[16] C. Rovelli, Black hole entropy from loop quantum gravity, Phys. Rev. Lett., 77, 3288 (1996)

[17] A. Ashtekar, J. Baez and K. Krasonv, Quantum geometry of isolated horizons and black hole entropy, Adv. Theor. Math. Phys., 4, 1 (2000)

[18] M. Bojowald, Loop quantum cosmology: Recent progress, Pramana 63 (2004) 765 [arXiv:gr-qc/0402053].

[19] K. Vandersloot, Hamiltonian constraint of loop quantum cosmology, Phys. Rev. D 71103506 (2005)

[20] M. Bojowald, Quantization ambiguities in isotropic quantum geometry, Class. Quant. Grav. 19 (2002) 5113, [arXiv:gr-qc/0206053].

[21] M. Bojowald, J. Lidsey, D. J. Mulryne, P. Singh and R. Tavakol,Inflationary Cosmology and Quantisation Ambiguities in Semi-Classical Loop Quantum Gravity, Phys. Rev. D 70, 043530 (2004).

[22] G. W. Gibbons, S. W. Hawking and J. M. Stewart, Nucl. Phys. B, 281, 736 (1986)

[23] S. Hollands and R. M. Wald, An alternative to inflation, Gen. Rel. Grav. 34 (2002) 2043 [arXiv:gr-qc/0205058].

[24] L. Kofman, A. Linde and V. F. Mukhanov, Inflationary theory and alternative cosmology, JHEP 0210 (2002) 057 [arXiv:hep-th/0206088].

[25] D. H. Coule, Remarks on initial conditions for scalar field driven inflationary models, arXiv:0706.0205 [gr-qc]. 
[26] S. W. Hawking, D. N. Page, Nucl. Phys. B 298, 789 (1988)

[27] W. R. Stoeger, G. F. R. Ellis and U. Kirchner Mon. Not. Roy. Astron. Soc. 347 (2004) 921.

[28] J. A. Peacock, Cosmological Physics, [Cambridge University Press, Cambridge (2002)].

[29] M. Bojowald, Inflation from quantum geometry, Phys. Rev. Lett. 89 (2002) 261301 [arXiv:gr-qc/0206054].

[30] S. Tsujikawa, P. Singh and R. Maartens, Class. Quant. Grav. 21 (2004) 5767. 